\title{
Efficacy and safety of vascular intervention combined with intravenous thrombolysis in treatment of acute intracranial arterial occlusion
}

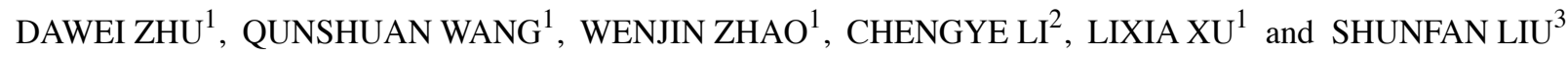 \\ ${ }^{1}$ Department of Medical Imaging, People's Hospital of Tongchuan; ${ }^{2}$ Department of Interventional Radiography, \\ Tongchuan Mining Bureau Central Hospital, Tongchuan, Shaanxi 727000; ${ }^{3}$ Department of Interventional Radiography, \\ Ninth Hospital of Xi'an, Xi'an, Shaanxi 710054, P.R. China
}

Received November 5, 2019; Accepted June 5, 2020

DOI: $10.3892 /$ etm.2020.9027

\begin{abstract}
Efficacy and safety of vascular intervention combined with intravenous thrombolysis (IVT) was investigated in the treatment of acute intracranial arterial occlusion (AIAO). Ninety-two patients with AIAO treated in People's Hospital of Tongchuan from January 2014 to February 2016 were enrolled in this retrospective study. Forty-two patients were treated with vascular intervention (control group), while another 50 patients were treated with vascular intervention combined with IVT (study group). They were observed in terms of the improvement of clinical efficacy after treatment, the comparison of complications after treatment, the National Institute of Health Stroke Scale (NIHSS) score after treatment, the modified Rankin Scale (mRS) score at 3 months after treatment, and the Mini-Mental State Examination (MMSE) score at 3 months after treatment. Compared with those in the control group, patients in the study group had statistically significantly higher marked effectiveness and statistically significantly lower ineffectiveness $(\mathrm{P}=0.018)$, and a statistically significantly higher overall effective rate $(\mathrm{P}=0.042)$. The NIHSS score in the study group was statistically significantly lower than that in the control group after treatment $(\mathrm{P}=0.001)$. There was a statistically significant difference between the two groups in the mRS score at 3 months after treatment $(Z=8.764, P>0.05)$. Compared with those in the control group, patients in the study group had a statistically significantly higher MMSE score after treatment, and a statistically significantly lower total incidence of postoperative complications $(\mathrm{P}=0.001)$.
\end{abstract}

Correspondence to: Dr Shunfan Liu, Department of Interventional Radiography, Ninth Hospital of Xi'an, 151 Erhuan East Road, Xi'an, Shaanxi 710054, P.R. China

E-mail:sbgm77@163.com

Key words: vascular intervention, intravenous thrombolysis, acute intracranial arterial occlusion, efficacy, adverse reactions
Vascular intervention combined with IVT has good efficacy and high safety in the treatment of AIAO, and the combination can statistically significantly improve patients' quality of life, so it has a good clinical application value.

\section{Introduction}

Cerebrovascular diseases are one of the major health problems in the world (1). According to Love and Miners (2), there were $\sim 1.6$ million patients with the diseases worldwide in 2016, most of whom were middle-aged and elderly. According to Kamat et al (3), increasing number of young people are developing the diseases, so it is predicted that young and middle-aged patients will account for $35 \%$ of patients of all ages by 2030 . Cerebrovascular diseases are extremely sudden, so many patients become disabled and die due to untimely rescue (4). These diseases are a research hotspot in clinical practice because of their high incidences and mortality. Main diseases among cerebrovascular diseases are cerebral ischemic disease with the infarction of middle cerebral artery or branch artery, accounting for $\sim 75 \%$ of all patients (5). Therefore, the timely and effective opening of occluded vessels as well as the recovery of cerebral tissue perfusion are crucial to treat acute intracranial arterial occlusion (AIAO). According to Powers et al (6), intravenous thrombolysis (IVT) is the first choice for the treatment of AIAO, but its therapeutic effect is unsatisfactory due to its low recanalization rate. Therefore, finding an effective therapeutic method for AIAO is very important.

Vascular intervention is the product of the iterative design and development process of evolving concepts in vascular biology and engineering (7). According to the study by Rogers and Edelman, the delivery of drugs near the implanted device may make the drugs exert their best effects (8). IVT, recommended by guidelines worldwide, is the first choice for the treatment of AIAO, but whether its combination with vascular intervention can treat the disease still need further study. Therefore, the efficacy and safety of the combination in the treatment of AIAO were explored in the present study, to provide accurate basis for the future treatment of the disease. 


\section{Patients and methods}

Clinical data. In this retrospective study, 92 patients with AIAO treated in People's Hospital of Tongchuan (Tongchuan, Chna) from January 2014 to February 2016 were enrolled. Forty-two patients were treated with vascular intervention (the control group), including 29 males and 13 females, aged $35-70$ years with an average age of 52.6 \pm 10.3 years. Fifty patients were treated with vascular intervention combined with IVT (study group), including 34 males and 16 females, aged 36-72 years, with an average age of $53.1 \pm 10.6$ years. The study was approved by the Ethics Committee of the People's Hospital of Tongchuan. Patients who participated in this study signed an informed consent and had complete clinical data.

\section{Inclusion and exclusion criteria}

Inclusion criteria. Patients diagnosed and treated in People's Hospital of Tongchuan; patients with complete general information; patients aged 35-75 years; patients with the educational background of primary school or above; patients who cooperated in the investigation; patients without intracranial hemorrhage detected by head CT; patients who or whose immediate family members signed the informed consent form.

Exclusion criteria. Patients who died during the treatment; patients complicated with other tumors; patients with physical disability; patients transferred to other hospitals; patients resistant or allergic to the drugs used in this study; patients with a history of intracranial hemorrhage including suspected subarachnoid hemorrhage; patients with a history of head injury in the past 3 months; patients with mental disorders, language dysfunction, or diseases affecting the results of this study.

Therapeutic schemes. Patients in the control group were treated with vascular intervention. After placed in a supine position, the patients were locally anesthetized and then intravenously injected with heparin $(2-3 \mathrm{mg} / \mathrm{kg})$, once every $2 \mathrm{~h}$ (half of the last dose). The lowest dose was maintained at $10 \mathrm{mg} / \mathrm{h}$. The arterial sheath was inserted through femoral artery using Seldinger method, and the $5 \mathrm{~F}$ catheter was used for brain angiography (iohexol was used as a contrast agent). The angiography was repeatedly performed to select the best work position. After the microcatheter was passed through the occluded blood vessel using directed acyclic graph and under the guidance of the micro-guide wire, the $4 \times 15 \mathrm{~mm}$ Solitaire $^{\mathrm{TM}}$ AB stent [no. SFDA(I) 20133465291] was placed in the occluded artery via the microcatheter and then released. After opened and maintained for more than $10 \mathrm{sec}$, the stent was retracted together with the microcatheter. The angiography after thrombectomy was performed to check whether the blood vessel was unobstructed, and multiple thrombectomy was conducted if necessary. The sheath was pulled out at $6 \mathrm{~h}$ after the operation. Patients in the study group were treated with vascular intervention combined with IVT. The steps of vascular intervention were as above, and alteplase (Boehringer Ingelheim Pharma GmbH \& Co. KG, item no. RK20180329n) and isotonic saline (100 IU+100 $\mathrm{ml}$ in total) were intravenously dripped for $30 \mathrm{~min}$. Changes in blood pressure and heart rate during the treatment were closely monitored.
Scoring criteria. The National Institute of Health Stroke Scale (NIHSS) was used for scoring (9). At 3 months after treatment, the modified Rankin Scale (mRS) score was used to assess the neurological function recovery of the patients to assess their prognoses (10). The Mini-Mental State Examination (MMSE) score was used to assess patients' cognitive function before treatment and 3 months after treatment.

Efficacy evaluation. Markedly effective outcome: symptoms disappeared and the patient's nerve function returned to normal. Effective outcome: Symptoms disappeared and the patient's nerve function improved. No effect, no improvement in the above indicators.

Follow-up. The patients were followed up for 3 months after operation, and their adverse reactions were recorded by telephone and outpatient medical records.

Outcome measures. Main outcome measures: The improvement of clinical efficacy after treatment was observed. Comparison of complications after treatment was carried out. Secondary outcome measures: The NIHSS score after treatment was recorded. The mRS score at 3 months after treatment and the MMSE score at 3 months after treatment were recorded.

Statistical analysis. In this study, SPSS 20.0 was used to statistically analyze the collected data. GraphPad 7 was used to plot figures. K-S test was used to analyze the distribution of measurement data. The data that conformed to normal distribution were expressed as mean \pm standard deviation (mean $\pm \mathrm{SD}$ ), analyzed by parametric tests, and represented by $\mathrm{Z}$. The comparison between groups was conducted by independent samples t-test, and the comparison within groups was conducted by paired t-test. Count data were expressed by rate (\%), analyzed by Chi-square test, and represented by $\chi^{2}$. $\mathrm{P}<0.05$, was accepted as a statistically significant difference.

\section{Results}

Clinical data. There was no statistically significant difference between the study and control groups in age, sex, body mass index (BMI), marital status, ethnicity, place of residence, smoking, NIHSS score (points) at admission, and MMSE score (points) at admission, which indicated comparability $(\mathrm{P}>0.05)$ (Table I).

Clinical efficacy. Compared with those in the control group, patients in the study group had statistically significantly higher marked effectiveness and statistically significantly lower ineffectiveness $(\mathrm{P}=0.018)$, without statistically significant difference in effectiveness between the two groups $(\mathrm{P}=0.224)$. The overall effective rate in the study group was statistically significantly higher than that in the control group ( $\mathrm{P}=0.042)$ (Table II).

NIHSS score after treatment. Before treatment, the NIHSS score was $13.03 \pm 4.21$ points in the study group and $13.01 \pm 3.08$ points in the control group. After treatment, the score was $9.01 \pm 1.22$ points in the study group and $10.85 \pm 2.71$ points in the control group. There was no 
Table I. Clinical data $[\mathrm{n}(\%)$, mean $\pm \mathrm{SD}]$

\begin{tabular}{|c|c|c|c|c|}
\hline Features & Study group $(n=50)$ & Control group $(n=42)$ & $\chi^{2}$ or t value & P-value \\
\hline Age (years) & $53.1 \pm 10.6$ & $52.6 \pm 10.3$ & 0.228 & 0.820 \\
\hline Sex & & & 0.012 & 0.914 \\
\hline Male & $34(68.00)$ & $29(69.05)$ & & \\
\hline Female & $16(32.00)$ & $13(30.95)$ & & \\
\hline BMI $\left(\mathrm{kg} / \mathrm{m}^{2}\right)$ & $22.26 \pm 0.37$ & $22.21 \pm 0.25$ & 0.744 & 0.459 \\
\hline Marital status & & & 0.068 & 0.794 \\
\hline Married & $47(94.00)$ & $40(95.24)$ & & \\
\hline Unmarried & $3(6.00)$ & $2(4.75)$ & & \\
\hline Ethnicity & & & 0.076 & 0.782 \\
\hline Han & $37(74.00)$ & $30(71.43)$ & & \\
\hline Ethnic minorities & $13(26.00)$ & $12(28.57)$ & & \\
\hline Place of residence & & & 0.007 & 0.934 \\
\hline City & $29(58.00)$ & $24(57.14)$ & & \\
\hline Countryside & $21(42.00)$ & $18(42.86)$ & & \\
\hline History of smoking & & & 0.024 & 0.877 \\
\hline Yes & $27(54.00)$ & $22(52.38)$ & & \\
\hline No & $23(46.00)$ & $20(47.62)$ & & \\
\hline NIHSS score (points) & $13.03 \pm 4.21$ & $13.01 \pm 3.08$ & 0.026 & 0.980 \\
\hline MMSE score (points) & $18.01 \pm 4.02$ & $18.03 \pm 3.01$ & 0.027 & 0.979 \\
\hline
\end{tabular}

NIHSS, National Institute of Health Stroke Scale; MMSE, Mini-Mental State Examination.

Table II. Efficacy.

\begin{tabular}{|c|c|c|c|c|c|}
\hline \multirow[b]{2}{*}{ Groups } & \multirow[b]{2}{*}{ No. of cases } & \multicolumn{3}{|c|}{ Efficacy $[\mathrm{n}(\%)]$} & \multirow[b]{2}{*}{ Effective rate $(\%)$} \\
\hline & & Markedly effective & Effective & Ineffective & \\
\hline Study group & 50 & $37(74.00)$ & $11(22.00)$ & $2(4.00)$ & 96.00 \\
\hline Control group & 42 & $21(50.00)$ & $14(33.33)$ & $7(16.67)$ & 83.00 \\
\hline$\chi^{2}$ value & & 5.643 & 1.482 & 4.150 & 4.150 \\
\hline P-value & & 0.018 & 0.224 & 0.042 & 0.042 \\
\hline
\end{tabular}

Chi-square test was used.

statistically significant difference between the two groups in the score before treatment $(\mathrm{P}=0.980)$. After treatment, the score in the study group was statistically significantly lower than that in the control group $(\mathrm{P}=0.001)$ (Fig. 1).

$m R S$ score at 3 months after treatment. The patients were followed up at 3 months after treatment. There was a statistically significant difference between the study and control groups in the mRS score at 3 months after treatment $(Z=8.764$, $\mathrm{P}>0.05$ ) (Table III).

MMSE score at 3 months after treatment. Before treatment, the MMSE score was $18.01 \pm 4.02$ points in the study group and $18.03 \pm 3.01$ points in the control group. After treatment, the score was $24.15 \pm 5.03$ points in the study group and
$20.35 \pm 5.12$ points in the control group. There was no statistically significant difference between the two groups in the score before treatment $(\mathrm{P}=0.179)$. After treatment, the score in the study group was statistically significantly higher than that in the control group ( $\mathrm{P}=0.001)$ (Fig. 1).

Complications after treatment. After treatment, the study group had 1 case of intracranial hypertension, 2 cases of dysphagia, 1 case of urinary incontinence, and 1 case of abnormal body temperature, while the control group had 3 cases of intracranial hypertension, 4 cases of dysphagia, 3 cases of urinary incontinence, and 2 cases of abnormal body temperature. The total incidence of postoperative complications in the study group was statistically significantly lower than that in the control group $(\mathrm{P}=0.022)$ (Table IV). 
A
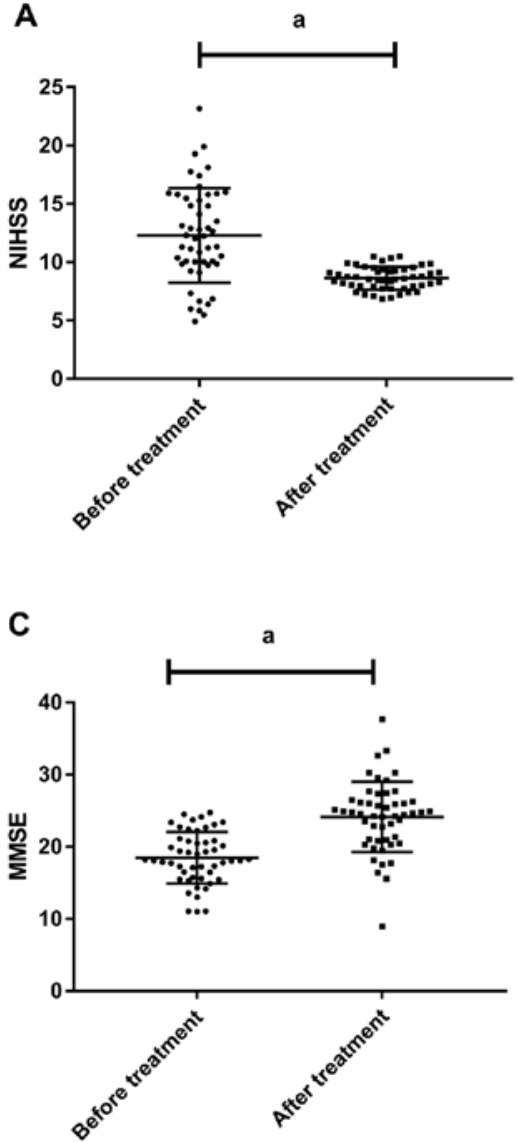
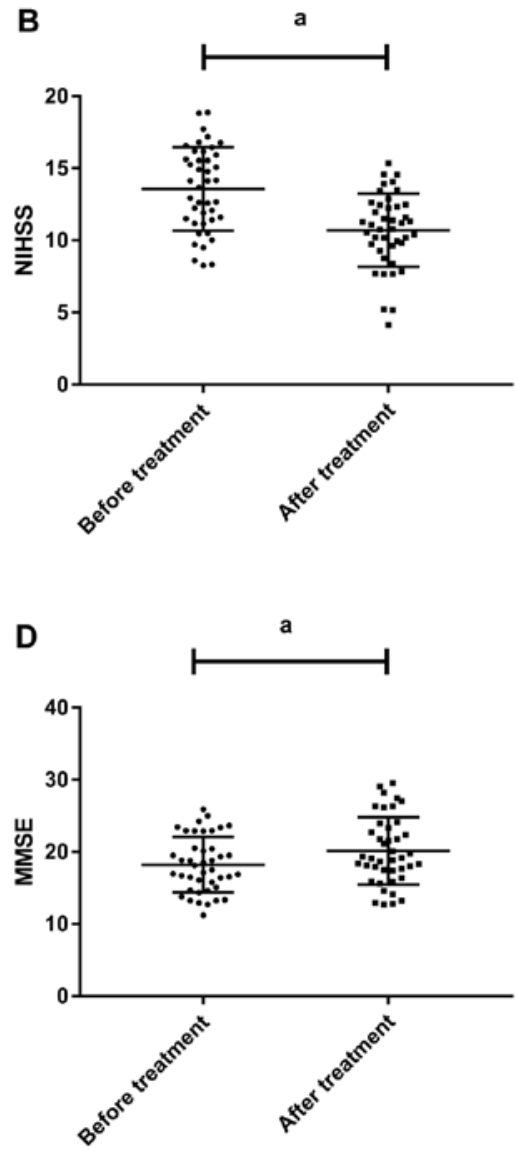

Figure 1. (A) In the study group, the NIHSS score was $13.03 \pm 4.21$ points before treatment and $9.01 \pm 1.22$ points after treatment, with a statistically significant difference between before and after treatment. (B) In the control group, the NIHSS score was $13.01 \pm 3.08$ points before treatment and $10.85 \pm 2.71$ points after treatment, with a statistically significant difference between before and after treatment. (C) In the study group, the MMSE score was $18.01 \pm 4.02$ points before treatment and $24.15 \pm 5.03$ points after treatment, with a statistically significant difference between before and after treatment. (D) In the control group, the MMSE score was $18.03 \pm 3.01$ points before treatment and $20.35 \pm 5.12$ points after treatment, with a statistically significant difference between before and after treatment. ${ }^{\mathrm{a}} \mathrm{P}<0.05$.

Table III. Comparison of the mRS score after treatment.

\begin{tabular}{lcccccccc}
\hline Groups & No. of cases & 0 point & 1 point & 2 points & 3 points & 4 points & 5 points & 6 points \\
\hline Study group & 50 & 0 & 37 & 10 & 1 & 2 & 0 & 0 \\
Control group & 42 & 0 & 21 & 6 & 5 & 3 & 7 & 0
\end{tabular}

Zero point indicates no symptom. One point indicates that the patient has symptoms but no functional defects. Two points indicate that the patient has mild disability. Three points indicate that the patient has moderate disability and needs some help. Four points indicate that the patient needs help in daily life. Five points indicate that the patient is unable to take care of himself and completely in need of help. Six points indicate patient death. mRS, modified Rankin Scale.

Table IV. Complications after treatment [n (\%)].

\begin{tabular}{|c|c|c|c|c|}
\hline Complications & Study group $(\mathrm{n}=50)$ & Control group $(n=42)$ & $\chi^{2}$ value & P-value \\
\hline Intracranial hypertension & $1(2.00)$ & $3(7.14)$ & & \\
\hline Pneumonia & $0(0.00)$ & $0(0.00)$ & & \\
\hline Dysphagia & $2(4.00)$ & $4(9.52)$ & & \\
\hline Urinary incontinence & $1(2.00)$ & $3(7.14)$ & & \\
\hline Acute renal failure & $0(0.00)$ & $0(0.00)$ & & \\
\hline Cardiac damage & $0(0.00)$ & $0(0.00)$ & & \\
\hline Abnormal body temperature & $1(2.00)$ & $2(4.76)$ & & \\
\hline Total & $5(10.00)$ & $12(28.56)$ & 5.226 & 0.022 \\
\hline
\end{tabular}




\section{Discussion}

The incidence of cerebrovascular diseases among the elderly is the highest, however, the pathophysiological mechanism of brain responding to cerebral ischemia in the elderly is still poorly understood (11). Old age is a major risk factor for the diseases and is associated with their increasing incidence and mortality $(12,13)$. In most developed countries, $\sim 87 \%$ of strokes are ischemic and originate from intracranial arterial occlusion (IAO), being the major cause of patient death and adult disability $(14,15)$. Therefore, it is urgent for clinicians to improve the condition of patients with IAO.

The pathogenesis of IAO is based on atherosclerotic plaque formation, Takayasu's arteritis, and vascular fibromuscular dysplasia (16). At present, patients with IAO are mainly treated by IVT and vascular intervention (17-19). According to Bracard et al (20), IVT with alteplase (a commonly used thrombolytic drug in clinical practice) alone cannot achieve the best therapeutic effect. Therefore, mechanical thrombectomy and IVT are used for the treatment to improve the patients' functional independence and to reduce their mortality. In this study, patients with AIAO were treated with vascular intervention combined with IVT, and the improvement of clinical efficacy and the safety of treatment were observed, to provide reference for the clinical treatment.

In this study, the clinical efficacy after treatment was first compared between the two groups. The results showed that compared with those in the control group, patients in the study group had statistically significantly higher marked effectiveness, statistically significantly lower ineffectiveness, and a statistically significantly higher overall effective rate. This shows that vascular intervention combined with IVT can increase the effective rate of treatment. NIHSS, one of the scales for evaluating the neurological function of patients with cerebrovascular diseases, can reflect the patients' neurological impairment and accurately determine their prognoses (21). There are currently few comparative studies on scoring systems for evaluating the severity and prognosis of neurological impairment in the patients. In our study, before treatment, there was no statistically significant difference between the two groups in the NIHSS score, while the score in the study group was statistically significantly lower than that in the control group after treatment. This suggests that vascular intervention combined with IVT can reduce the patients' neurological deficits. The mRS can measure patients' neurological function recovery (10), and psychological and physical factors affecting the recovery are included. The scale includes 6 grades (from 0 to 5), with 0 indicating no symptom and 5 points indicating severe disability. The patients in this study were followed up at 3 months after treatment, and the mRS score was used to judge the neurological function recovery. The mRS score in the study group was statistically significantly better than that in the control group. Additionally, there was no difference between the two groups in the MMSE score before treatment, but the score in the study group was higher than that in the control group after treatment. This reveals that vascular intervention combined with IVT can better improve the prognosis than IVT alone. Finally, the complications at 3 months after treatment were observed. Patients in the two groups had no pneumonia, acute renal failure, or cardiac damage, while the incidence rate of intracranial hypertension, dysphagia, urinary incontinence, and abnormal body temperature in the study group was lower than those in the control group; the total incidence of complications in the study group was also lower than that in the control group. This well illustrates the prognostic effect and safety of vascular intervention combined with IVT.

In the present study, through the above research, the clinical efficacy of vascular intervention combined with IVT in the treatment of patients with AIAO was preliminarily proven. However, there are still limitations. Firstly, whether the treatment rate can be increased by changing the dosage was not fully investigated. Secondly, rat experiments and long-term follow-up were not conducted. We will carry out more in-depth experimental analysis and long-term follow-up as soon as possible.

In conclusion, vascular intervention combined with IVT has good efficacy and high safety in the treatment of AIAO, and the combination can statistically significantly improve the patients' quality of life, so it has a good clinical application value.

\section{Acknowledgements}

Not applicable.

\section{Funding}

No funding was received.

\section{Availability of data and materials}

The datasets used and/or analyzed during the current study are available from the corresponding author on reasonable request.

\section{Authors' contributions}

DZ wrote the manuscript. DZ and QW conceived and designed the study. QW and WZ were responsible for the collection and analysis of the experimental data. CL and LX interpreted the data and drafted the manuscript. SL was responsible for the efficacy evaluation and the patients' follow-up. All authors read and approved the final manuscript.

\section{Ethics approval and consent to participate}

The study was approved by the Ethics Committee of the People's Hospital of Tongchuan (Tongchuan, China). Patients who participated in this study signed an informed consent and had complete clinical data.

\section{Patient consent for publication}

Not applicable.

\section{Competing interests}

The authors declare that they have no competing interests. 


\section{References}

1. Mozaffarian D, Benjamin EJ, Go AS, Arnett DK, Blaha MJ, Cushman M, Das SR, de Ferranti S, Després JP, Fullerton HJ, et al; Writing Group Members; American Heart Association Statistics Committee; Stroke Statistics Subcommittee: Heart disease and stroke statistics-2016 update: A report from the American Heart Association. Circulation 133: e38-e360, 2016.

2. Love S and Miners JS: Cerebrovascular disease in ageing and Alzheimer's disease. Acta Neuropathol 131: 645-658, 2016.

3. Kamat PK, Vacek JC, Kalani A and Tyagi N: Homocysteine induced cerebrovascular dysfunction: A link to Alzheimer's disease etiology. Open Neurol J 9: 9-14, 2015.

4. Lahousse L, Tiemeier H, Ikram MA and Brusselle GG: Chronic obstructive pulmonary disease and cerebrovascular disease: A comprehensive review. Respir Med 109: 1371-1380, 2015.

5. Drandley TD and Floras JS (eds): Sleep Apnea: Implications in Cardiovascular and Cerebrovascular Disease. CRC Press, Florida, 2016.

6. Powers WJ, Rabinstein AA, Ackerson T, Adeoye OM, Bambakidis NC, Becker K, Biller J, Brown M, Demaerschalk BM, Hoh B, et al; American Heart Association Stroke Council: 2018 Guidelines for the early management of patients with acute ischemic stroke:a guideline for healthcare professionals from the American Heart Association/American Stroke Association. Stroke 49: e46-e110, 2018.

7. Nakamura K, Keating JH and Edelman ER: Pathology of endovascular stents. Interv Cardiol Clin 5: 391-403, 2016.

8. Rogers $\mathrm{C}$ and Edelman ER: Endovascular stent design dictates experimental restenosis and thrombosis. Circulation 91: 2995-3001, 1995.

9. Ortiz GA and Sacco R: National Institutes of Health Stroke Scale (nihss). Wiley Statistics Reference Online, 2014.

10. Sulter G, Steen C and De Keyser J: Use of the Barthel index and modified Rankin scale in acute stroke trials. Stroke 30: $1538-1541,1999$

11. Di Napoli M and Shah IM: Neuroinflammation and cerebrovascular disease in old age: A translational medicine perspective. J Aging Res 2011: 857484, 2011.
12. Modrego PJ, Pina MA and Lerín FJ: The impact of ageing on stroke subtypes, length of stay and mortality: Study in the province of Teruel, Spain. Acta Neurol Scand 108: 435-442, 2003.

13. Saposnik G, Cote R, Phillips S, Gubitz G, Bayer N, Minuk J and Black S; Stroke Outcome Research Canada (SORCan) Working Group: Stroke outcome in those over 80: A multicenter cohort study across Canada. Stroke 39: 2310-2317, 2008.

14. Feigin VL: Stroke epidemiology in the developing world. Lancet 365: 2160-2161, 2005.

15. Feigin V and Hoorn SV: How to study stroke incidence. Lancet 363: 1920-1921, 2004.

16. DeBaun MR, Armstrong FD, McKinstry RC, Ware RE, Vichinsky E and Kirkham FJ: Silent cerebral infarcts: A review on a prevalent and progressive cause of neurologic injury in sickle cell anemia. Blood 119: 4587-4596, 2012.

17. Emberson J, Lees KR, Lyden P, Blackwell L, Albers G, Bluhmki E, Brott T, Cohen G, Davis S, Donnan G, et al; Stroke Thrombolysis Trialists' Collaborative Group: Effect of treatment delay, age, and stroke severity on the effects of intravenous thrombolysis with alteplase for acute ischaemic stroke: A meta-analysis of individual patient data from randomised trials. Lancet 384: 1929-1935, 2014.

18. Guillan M, Alonso-Canovas A, Garcia-Caldentey J, SanchezGonzalez V, Hernandez-Medrano I, Defelipe-Mimbrera A Matute MC, Alonso-Arias MA, Alonso de Leciñana M and Masjuan J: Off-label intravenous thrombolysis in acute stroke. Eur J Neurol 19: 390-394, 2012.

19. Göthe F, Enache D, Wahlund LO, Winblad B, Crisby M, Lökk J and Aarsland D: Cerebrovascular diseases and depression: Epidemiology, mechanisms and treatment. Panminerva Med 54: 161-170, 2012.

20. Bracard S, Ducrocq X, Mas JL, Soudant M, Oppenheim C, Moulin T and Guillemin F; THRACE investigators: Mechanical thrombectomy after intravenous alteplase versus alteplase alone after stroke (THRACE): A randomised controlled trial. Lancet Neurol 15: 1138-1147, 2016.

21. Goldstein LB, Bertels C and Davis JN: Interrater reliability of the NIH stroke scale. Arch Neurol 46: 660-662, 1989. 